\title{
A Describing Function for Resonantly Commutated H-Bridge Inverters
}

\author{
H. Isaac Sewell, David A. Stone, and Chris M. Bingham, Member, IEEE
}

\begin{abstract}
The paper presents the derivation of a describingfunction to model the dynamic behavior of a metal oxide semiconductor field effect transistor-based, capacitively commutated H-bridge, including a comprehensive explanation of the various stages in the switching cycle. Expressions to model the resulting input current, are also given. The derived model allows the inverter to be accurately modeled within a control system simulation over a number of utility input voltage cycles, without resorting to computationally intensive switching-cycle level, time-domain SPICE simulations. Experimental measurements from a prototype $\mathrm{H}$-bridge inverter employed in an induction heating application, are used to demonstrate a high degree of prediction accuracy over a large variation of load conditions is possible using the simplified model.
\end{abstract}

Index Terms-Metal oxide semiconductor field effect transistor (MOSFET)-based capacitively commutated H-bridge, switching cycle.

$\mathrm{C}_{1} \quad$ Capacitor across top-side, load commutated

$\mathrm{C}_{2} \quad$ Capacitor across top-side, PWM-controlled

$\mathrm{C}_{3} \quad$ Capacitor across bottom-side, load

$\mathrm{C}_{4} \quad$ commutated power switch.

PWM-controlled power switch.

$\left\{C_{1} \ldots C_{4}\right\} \quad$ Capacitance of $\left\{\mathrm{C}_{1} \ldots \mathrm{C}_{4}\right\}$.

$C_{\text {comm }}$

$C_{\text {comm(PWM) }}^{\prime}$

$C_{\text {comm(Load) }}^{\prime}$

$C_{\text {oss }}$

$\mathrm{D}_{1}$

$\mathrm{D}_{2}$

$\mathrm{D}_{3}$

$\mathrm{D}_{4}$

$F_{c}\left(V_{\mathrm{dc}}\right)$

\section{NOMENCLATURE} power switch.

$f_{s}$

$I_{\mathrm{cos}}$

$I_{\mathrm{dc}}$

$I_{\text {out }}$

$I_{\text {sin }}$

$i_{\text {out }}$

$q_{1}$

$q_{2}$

$q_{3}$

$q_{4}$

$q_{5}$

$q_{6}$

$q_{7}$

$q_{8}$

Capacitance involved in the

commutation of the switch.

Equivalent capacitance involved in the

commutation of the PWM leg.

Equivalent capacitance involved in the commutation of the load-commutated leg.

Parasitic capacitance across the device.

Antiparallel diode across top-side, load

commutated power switch.

Antiparallel diode across top-side,

PWM-controlled power switch.

Antiparallel diode across bottom-side, load commutated power switch.

Antiparallel diode across bottom-side,

PWM-controlled power switch.

Function relating the charge held in the parasitic capacitance of the MOSFET to the dc-link voltage.

$R_{d}$

$R_{\text {on }}$

$T_{\text {on }}$

$V_{d}$

$V_{\mathrm{dc}}$

$V_{t}$

Manuscript received January 27, 2003; revised December 11, 2003. Recommended by Associate Editor B. Fahimi.

The authors are with the Department of Electronic and Electrical Engineering, Sheffield S1 3JD, U.K. (e-mail: d.a.stone@sheffield.ac.uk).

Digital Object Identifier 10.1109/TPEL.2004.830081

Switching frequency.

"quadrature" component of the output current vector.

Input current to the H-Bridge.

Magnitude of the equivalent sinusoidal

representation of the output current.

"in-phase" component of the output current vector."

Time-domain representation of output current from the H-Bridge.

Charge transferred from the dc-link to the H-bridge during period 1 .

Charge transferred form the dc-link to the

H-bridge during period 2.

Charge transferred form the dc-link to the

$\mathrm{H}$-bridge during period 3 .

Charge transferred form the dc-link to the

$\mathrm{H}$-bridge during period 4.

Charge transferred form the dc-link to the

$\mathrm{H}$-bridge during period 5 .

Charge transferred form the dc-link to the

H-bridge during period 6 .

Charge transferred form the dc-link to the

H-bridge during period 7 .

Charge transferred form the dc-link to the

$\mathrm{H}$-bridge during period 8 .

$\mathrm{SW}_{1}$

$\mathrm{SW}_{3}$

$\mathrm{SW}_{2}$

$\mathrm{SW}_{4}$

$q_{\text {com }}$

$q_{\text {com limit }}$

On-state resistance of the diode.

On-state resistance of the switch.

Top-side, load commutated power switch.

Bottom-side, load commutated power switch.

Top-side, PWM-controlled power switch.

Bottom-side, PWM-controlled power switch.

On-time of the switch.

Charge stored in the total leg capacitance.

Angle at which the commutation cycle

finishes as the opposing switch turns on.

Time-domain representation of the generic leg

voltage during the time that the switches'

antiparallel diodes are carrying the output

current.

$V_{\text {diode }}$

dc-link voltage.

Forward voltage of the diode.

Voltage across the switch at the point of

turn-off.

$V_{\text {tr }}$

$V_{\text {Leg }}$

Forward voltage of switch.

FMA equivalent representation of the leg voltage.

Time-domain representation of the generic leg voltage during the time that the commutation capacitors are carrying the output current. 
$v_{\text {cond }} \quad$ Time-domain representation of the generic leg voltage during the time that the switches are carrying the output current.

$v_{\mathrm{ds}} \quad$ Voltage across the drain and source terminals of the MOSFET switch.

$v_{\mathrm{LC}} \quad$ Time-domain representation of the load commutated leg voltage.

$v_{\text {Leg }} \quad$ Time-domain representation of the generic leg voltage.

$v_{\mathrm{PWM}}$

Time-domain representation of

PWM-controlled leg voltage.

$v_{\text {out }} \quad$ Time-domain representation of output voltage from the H-Bridge.

$v_{\text {to }} \quad$ Time-domain representation of the generic leg voltage during the time that the switches are turning off.

$\theta_{1} \quad$ Angle at which the switch starts to turn off.

$\theta_{2} \quad$ Angle at which the switch completes turn-off.

$\theta_{3} \quad$ Angle at which the commutation finishes.

$\theta_{d} \quad$ Phase of the equivalent sinusoidal representation of the output current.

$\alpha_{1} \quad$ Angle at which $\mathrm{SW}_{4}$ starts to turn off.

$\alpha_{2} \quad$ Angle at which $\mathrm{SW}_{4}$ completes turn-off.

$\alpha_{3} \quad$ Angle at which the commutation of $\mathrm{SW}_{4}$ finishes.

$\beta_{1} \quad$ Angle at which $\mathrm{SW}_{1}$ starts to turn off.

$\beta_{2} \quad$ Angle at which $\mathrm{SW}_{1}$ completes turn-off.

$\beta_{3} \quad$ Angle at which the commutation of $\mathrm{SW}_{1}$ finishes.

\section{INTRODUCTION}

$\mathbf{T}$ HE MOST common high-frequency inverter circuit employed in an industrial environment consists of a capacitively commutated metal oxide semiconductor field effect transistor (MOSFET)-based H-bridge, a dc-link smoothing filter, Fig. 1, together with monitoring and feedback electronics. For high-frequency applications, the basic $\mathrm{H}$-bridge is often augmented with capacitors $\left(\mathrm{C}_{1} \ldots \mathrm{C}_{4}\right)$ in parallel with the power switches, to facilitate zero-voltage commutation of the inverter legs; a feature that has been shown to be advantageous in both IGBT and MOSFET-based bridges [1] since it allows high efficiency operation with very low switching loss. It also permits some control of $d v_{\text {out }} / d t$ at the output, thereby mitigating EMC problems. However, the incorporation of commutation capacitors has significant impact on the dynamic operation of the circuit, as the bridge commutation period becomes a significant proportion of the switching period. This substantially increases the complexity of models that can accurately predict circuit behavior, since they are required to describe the output voltage characteristics during the commutation periods, when the commutation capacitors support the output current. Although modeling the operation of low-power $L C C$ resonant converters with half-bridge switch networks [3] has been addressed with some success, the dynamic effects of commutation components in a full $\mathrm{H}$-bridge, for high power systems, remains outstanding.

Here then, the complex commutation effects within the $\mathrm{H}$-bridge inverter are described, along with time-domain and static performance characteristics. From this, a describingfunction to model the input/output characteristics of inverter

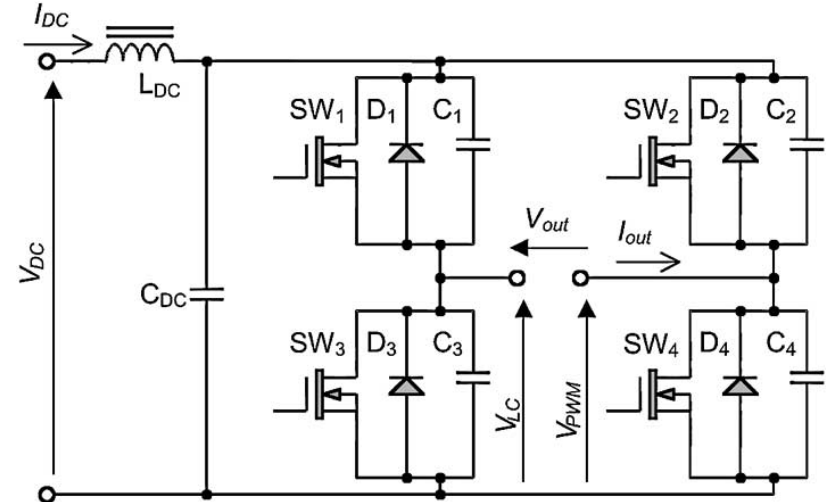

Fig. 1. Inverter circuit.

operation, is derived, and subsequently employed to predict system output current against PWM duty, thereby providing a macro-model of the H-bridge; a feature necessary to accurately, and rapidly, model an inverter within a control system simulation, for instance. Indeed, the proposed model typically executes some 10000 times faster than component-based simulation packages such as Spice. Additionally, the resulting model is sufficiently detailed to provide enhanced predictions of efficiency throughout the circuit, and facilitate optimized design and performance sensitivity results with respect to component values and tolerances. To provide a practical focus to the paper, application of the presented techniques is considered for modeling a $2.5-\mathrm{kW}$ inverter employed in an induction heating system.

\section{CiRCUit OpERATION}

The preferred use of high switching frequencies to reduce the size of reactive system components (or to achieve specific heating patterns in the case of induction heating), means that device switching can become the most significant loss mechanism within the inverter. Low-loss commutation strategies are therefore desirable, the most effective being zero-voltage commutation [2].

Since PWM is a requirement for control of power to the load, only one leg of the inverter $\left(\mathrm{SW}_{1}\right.$ and $\mathrm{SW}_{3}$, for instance) can switch at the zero-crossing times of the load current, while the remaining leg must commutate appropriately to provide the effective duty-cycle at the output, under zero-voltage commutation. This facilitates controlled power transfer without additional power preprocessing stages. To minimize switching losses in the fixed, "load commutated" leg, $\mathrm{SW}_{1}$ and $\mathrm{SW}_{3}$ are turned off as the output current is about to pass through zero. Although this appears to utilize zero current commutation, the devices are, in fact, commutated under reduced voltage by virtue of the presence of the commutation capacitors $\mathrm{C}_{1}$ and $\mathrm{C}_{3}$. This combination of low current and low voltage at the switching instant significantly reduces switching losses, and constitutes operation under optimal conditions described by Dede et al. [2]. However, since the output voltage is controlled using pulse width modulation (PWM), $\mathrm{SW}_{2}$, and $\mathrm{SW}_{4}$ cannot be load commutated in the same manner. Consequently, capacitors $\mathrm{C}_{2}$ and $\mathrm{C}_{4}$ are included to allow zero-voltage commutation 


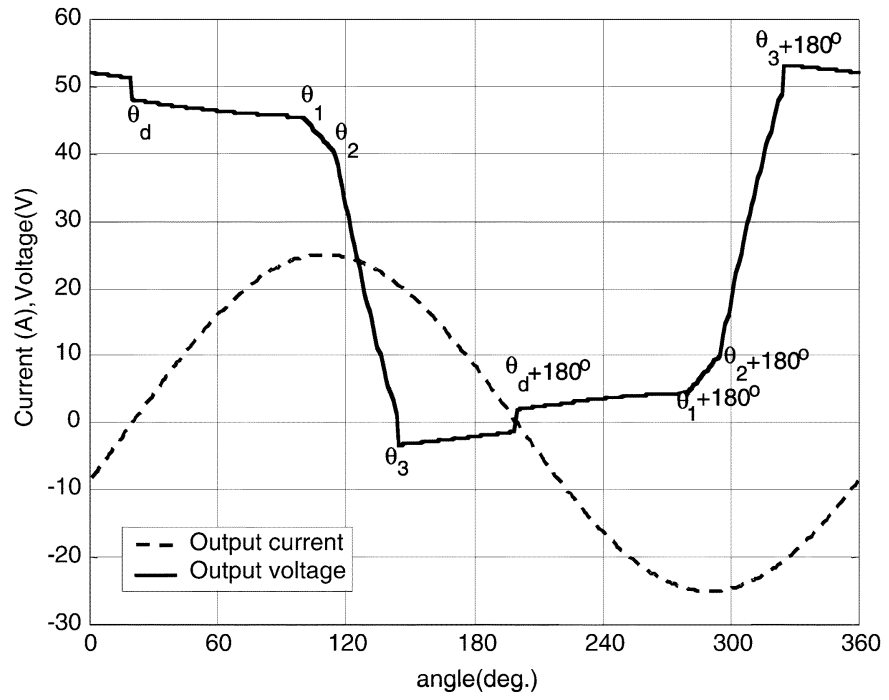

Fig. 2. Inverter leg voltage and load current.

of $\mathrm{SW}_{2}$ and $\mathrm{SW}_{4}$, thereby reducing the switching losses and decreasing the $d v / d t$ across the switches. $\mathrm{C}_{2}$ and $\mathrm{C}_{4}$ are larger than $\mathrm{C}_{1}$ and $\mathrm{C}_{3}$, as the potential instantaneous currents they are to handle are higher, given that the instantaneous maximum in the output current may occur during the commutation event. The resulting inverter output voltage is then given by the difference between the inverter bridge-leg voltages (1) (see Fig. 1)

$$
v_{\mathrm{out}}=v_{\mathrm{LC}}-v_{\mathrm{PWM}}
$$

The characteristics of both $v_{\mathrm{LC}}$ and $v_{\mathrm{PWM}}$ may be analyzed independently and subsequently combined to give the overall describing function for the inverter output voltage.

\section{Generic Model of LEG Voltage}

Typical leg-voltage $\left(v_{\text {Leg }}\right)$ and current $\left(i_{\text {out }}\right)$ waveforms during steady-state operation of the inverter are shown in Fig. 2, where the output of the leg is loaded by a sinusoidal current sink [see Fig. 3(a)] and time has been normalized based on the switching period to provide waveforms as a function of angle. A describing function modeling the behavior of the leg of the inverter is obtained by considering the piecewise time-domain operation of the inverter between the various mode transition angles $\theta_{n}$ shown in Fig. 2.

The phase reference for the voltage, $\theta=0$, coincides with the turn-on of the upper top-left switch, $\mathrm{SW}_{1}$ in Fig. 1, and occurs while the anti-parallel diode is conducting. The anti-parallel diode ceases to conduct at $\theta_{d}$, which also defines the phase-shift between the output voltage and the sinusoidal output current, described by

$$
i_{\text {out }}(\theta)=I_{\text {out }} \sin \left(\theta-\theta_{d}\right)
$$

During the period $\theta_{d} \rightarrow \theta_{1}, \mathrm{SW}_{1}$ is forward conducting and the leg voltage during this time, $v_{\text {cond }}$, is modeled as a quasisquare (a)

(b)
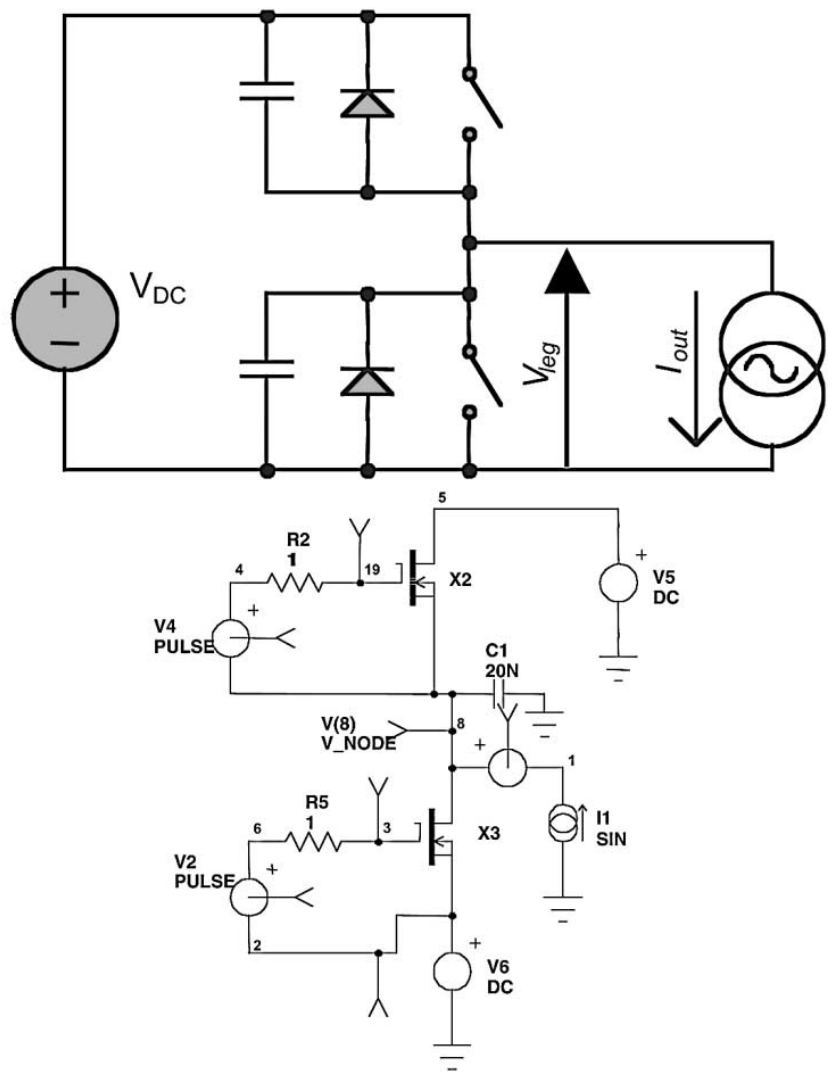

Fig. 3. (a) Generic inverter leg model and (b) Spice model circuit.

wave modified by a sinusoidal perturbation to account for conduction resistance of the switching device

$$
\begin{aligned}
v_{\text {Leg }}(\theta) & =v_{\text {cond }}(\theta) \\
& = \begin{cases}V_{\mathrm{DC}}-V_{\mathrm{tr}}-i_{\text {out }}(\theta) R_{\mathrm{on}} & \theta=\theta_{d} \ldots \theta_{1} \\
V_{\mathrm{tr}}-i_{\text {out }}(\theta) R_{\text {on }} & \theta=\pi+\theta_{d} \ldots \pi+\theta_{1}\end{cases}
\end{aligned}
$$

where the forward-conduction voltage drop of the device, $V_{\mathrm{tr}}$, is included to allow for either reverse conduction blocking diodes or the use of IGBTs. Reverse blocking diodes may be used when several MOSFETs are paralleled, since the rate-of-change of diode forward voltage with temperature does not encourage current sharing of the devices during the diode conduction phase. Also given in (3) is the voltage characteristic during the phase period $\theta=\pi+\theta_{d}$ to $\pi+\theta_{1}$ when the opposite switch in the bridge leg is conducting ( $\mathrm{SW}_{3}$ in Fig. 1).

During the period $\theta_{1} \rightarrow \theta_{2}$, the voltage across $\mathrm{SW}_{1}$ rises to $V_{t}$, the "terminal" voltage of the switch. Extensive practical measurements show that this rise can be modeled as being essentially linear. The leg voltage, $V_{\text {to }}$, therefore reduces accordingly during this time, as described by (4), shown at the bottom of the next page. The leg voltage during the complementary time, when $\mathrm{SW}_{3}$ carries current, is also given in (4).

During the period when commutation occurs, $\theta_{2} \rightarrow \theta_{3}$, the current from the resonant load charges the capacitance across the switches. The total capacitance consists of the nonlinear parasitic drain-source capacitance $\left(C_{\mathrm{oss}}\right)$, Fig. 4 , of both the transistor that is turning off and its complimentary paring, and any 


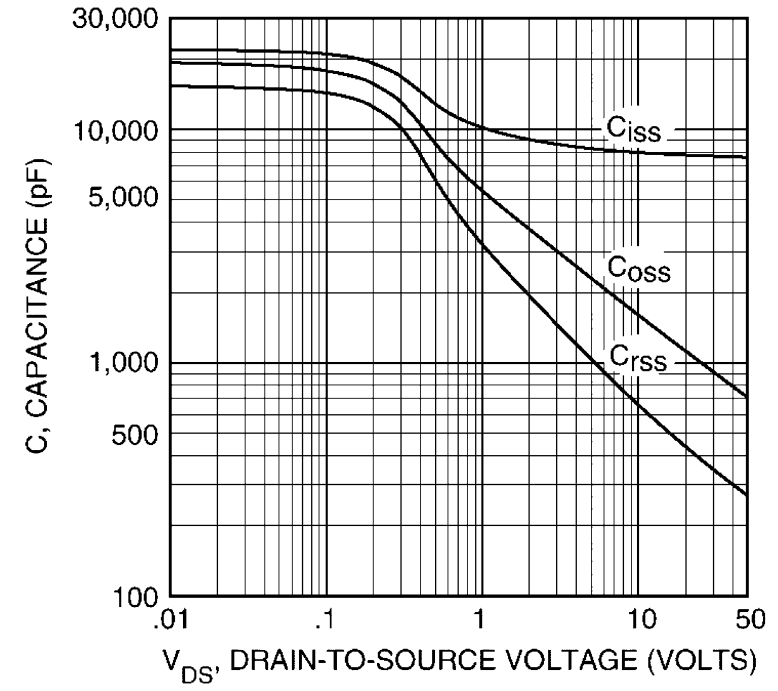

Fig. 4. Voltage dependency of MOSFET parasitic capacitances (reproduced courtesy of Advanced Power Technology: APT5010LVR).

additional component capacitance connected across the transistor. At low voltages, the drain-source capacitance dominates, while the commutation capacitance dominates at high voltages.

Since $\mathrm{SW}_{1}$ can be considered to be 'off' during this phase, all load current flows into the commutation capacitors and the parasitic drain-source capacitances. As the parasitic capacitors are a nonlinear function of applied voltage, the calculation of the commutation angle can be simplified by assuming $\mathrm{SW}_{1}$ turns off instantaneously, and the commutation capacitance is charged until it reaches the applied dc-link voltage. The stored charge is the integral of the output current, (5), taken over the commutation period, as given in

$$
i_{\text {out }}(t)=I_{\text {out }} \sin \left(2 \pi f_{s} t+\theta_{d}\right)
$$

$$
q_{\mathrm{com}}=\frac{I_{\mathrm{out}}}{2 \pi f_{s}} \int_{\theta_{2}}^{\theta_{3}} \sin \left(\theta+\theta_{d}\right) d \theta
$$

which can be solved for $\theta_{3}$

$$
\theta_{3}=\pi+\theta_{d}-\cos ^{-1}\left(\frac{2 \pi f_{s} q_{\mathrm{com}}}{I_{\text {out }}}-\cos \left(\theta_{2}-\theta_{d}\right)\right) .
$$

By assuming a sinusoidal leg current, and noting the voltage is $\pi / 2$ radians out of phase with the current, and of the same frequency, the output voltage can be written as (8), shown at the bottom of the page. Finally, between $\theta=\theta_{3}$ and $\theta=$ $\pi+\theta_{d}$, the voltage across $\mathrm{SW}_{1}$ exhibits a similar characteristic to the switch conduction period, differing only in the polarity of the offset. In particular, if the diode resistance $R_{d}$ is equal to the switch on-state resistance, $R_{\mathrm{tr}}$, and the conducting voltage drop across the diode, $V_{\text {diode }}$, is equal to the switch on-state voltage drop, $V_{\mathrm{tr}}$, then $v_{d}(\theta)=v_{\text {cond }}(\theta)$. In the more general case however, the output voltage is described by (9), shown at the bottom of the page. Equations (2)-(9), therefore, provide a generic piecewise description of the steady-state behavior of the inverter under the rather mild assumptions that 1) diode reverse recovery does not significantly influence the behavior of the inverter/load combination, 2) instantaneous output current remains positive during the period $\theta=\theta_{1} \rightarrow \pi$, and 3) the switch rather than the diode carries the current in the period $\theta=0 \rightarrow \theta_{1}$. The latter two conditions hold for a MOSFET, providing the magnitude of the instantaneous device current is less than that required to create a body-resistance voltage-drop of sufficient value to forward bias the internal anti-parallel diode into its conducting state.

To show the validity of the presented piecewise model of the inverter switching characteristics, a SPICE-based model of the inverter leg employing International Rectifier IRFPS40N50L MOSFET devices is used, Fig. 3(b). A sinusoidal current source is included to represent the effects of the resonant output load.

$v_{\mathrm{Leg}}=v_{\mathrm{to}}(\theta)=\left\{\begin{array}{l}V_{\mathrm{DC}}-V_{\mathrm{tr}}-R_{\mathrm{on}} I_{\mathrm{out}} \sin \left(\theta_{1}-\theta_{d}\right)-\left(\left(\theta-\theta_{1}\right) \frac{V_{t}-V_{\mathrm{tr}}-R_{\mathrm{on}} I_{\mathrm{out}} \sin \left(\theta_{1}-\theta_{d}\right)}{\theta_{2}-\theta_{1}}\right) \\ V_{\mathrm{tr}}+R_{\mathrm{on}} I_{\mathrm{out}} \sin \left(\theta_{1}-\theta_{d}\right)+\left(\left(\theta-\theta_{1}-\pi\right) \frac{V_{t}-V_{\mathrm{tr}}-R_{\mathrm{on}} I_{\mathrm{out}} \sin \left(\theta_{1}-\theta_{d}\right)}{\theta_{2}-\theta_{1}}\right) \quad \theta=\theta_{1}+\pi \ldots \theta_{2}+\pi\end{array}\right.$

$$
v_{\mathrm{Leg}}=v_{\mathrm{com}}(\theta)= \begin{cases}V_{\mathrm{DC}}-V_{d}-\frac{I_{\mathrm{out}}}{2 \pi f_{s} C_{\mathrm{comm}}} \cos \left(\theta_{d}-\theta_{2}\right)+\frac{I_{\mathrm{out}}}{2 \pi f_{s} C_{\mathrm{comm}}} \cos \left(\theta-\theta_{d}\right) & \theta=\theta_{2} \ldots \theta_{3} \\ V_{d}-\frac{I_{\mathrm{out}}}{2 \pi f_{s} C_{\mathrm{comm}}} \cos \left(\theta_{d}+\pi-\theta_{2}\right)+\frac{I_{\mathrm{out}}}{2 \pi f_{s} C_{\mathrm{comm}}} \cos \left(\theta-\theta_{d}\right) & \theta=\theta_{2}+\pi \ldots \theta_{3}+\pi\end{cases}
$$

$$
v_{\text {Leg }}=v_{d}(\theta)= \begin{cases}-V_{\text {diode }}-I_{\text {out }} \sin \left(\theta-\theta_{d}\right) R_{d} & \theta=\theta_{3} \ldots \pi+\theta_{d} \\ V_{\mathrm{DC}}+V_{\text {diode }}-I_{\text {out }} \sin \left(\theta-\theta_{d}\right) R_{d} & \theta=\theta_{3}+\pi \ldots 2 \pi+\theta_{d}\end{cases}
$$


(a)

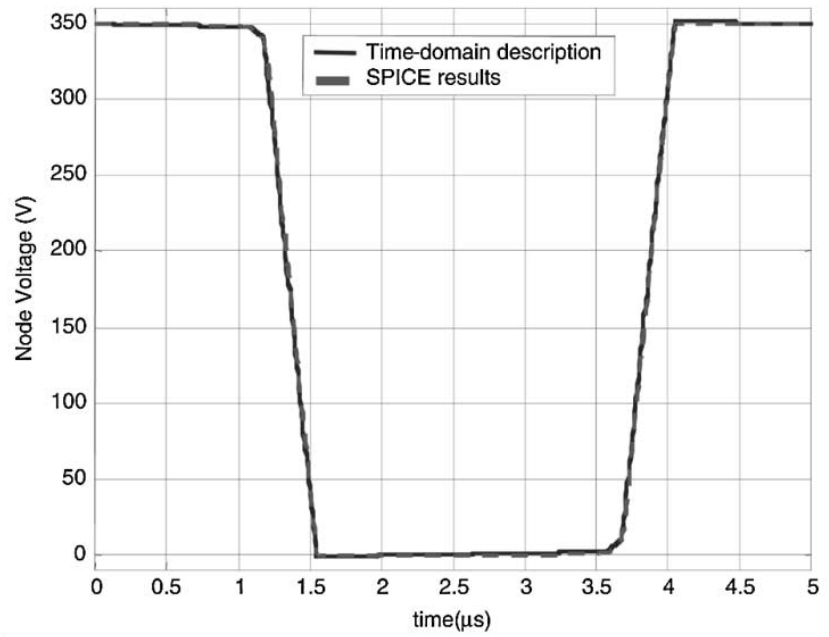

(b)

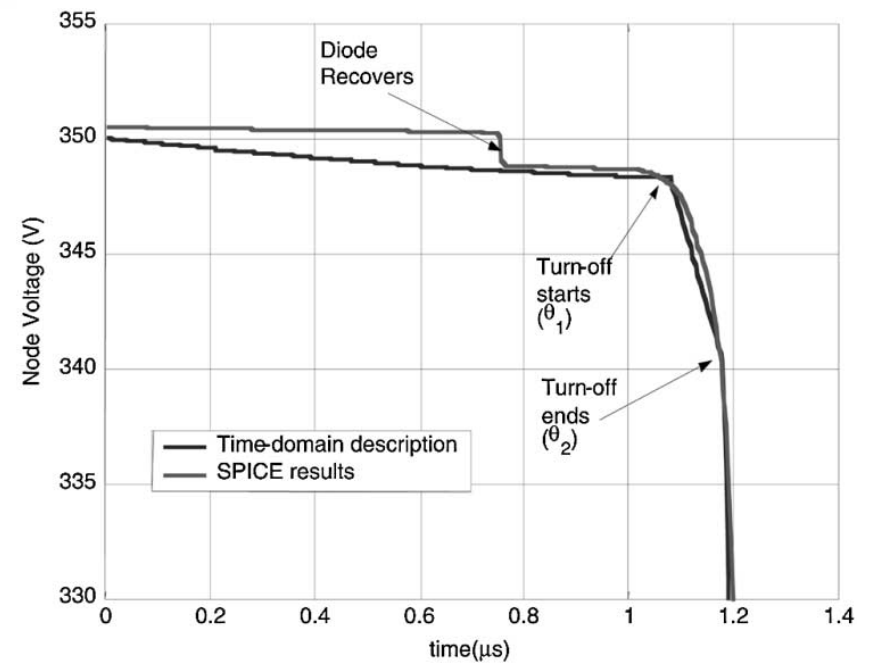

Fig. 5. (a) Switching cycle and (b) detail of diode recovery and transistor turn-off.

A comparison of results from time-domain SPICE simulations, with the predicted behavior of the inverter [from (3)-(9)], is shown in Fig. 5(a), and indicates good correspondence is achievable during the majority of the cycle. Fig. 5(b) shows a magnified view to indicate the typical influence of diode recovery, along with details of the transistor turn-off period. It can be seen that while there is a small difference in the shape of the output voltage waveforms, the times at which the turn-off begins and ends, and the voltages at which these occur, are predicted accurately by the model. \{Note: the SPICE results show the diode actually providing energy into the system during recovery. This is not an appropriate characteristic and is therefore not included in the piecewise time-domain model $\}$.

\section{DESCRIBING FunCTION OF GENERIC INVERTER LEG}

To derive the fundamental mode approximation (FMA) describing-function of a generic inverter leg, and investigate the domain of applicability of the proposed model, operation of the circuit [Fig. 3(b)] is considered for various switch turn-off angles, $\theta_{1}$, between $\theta_{1}=\theta_{d} \rightarrow \pi$. The describing function of the leg is initially obtained by taking the Real and Imaginary components of the fundamental from the FFT of the resulting time-do-

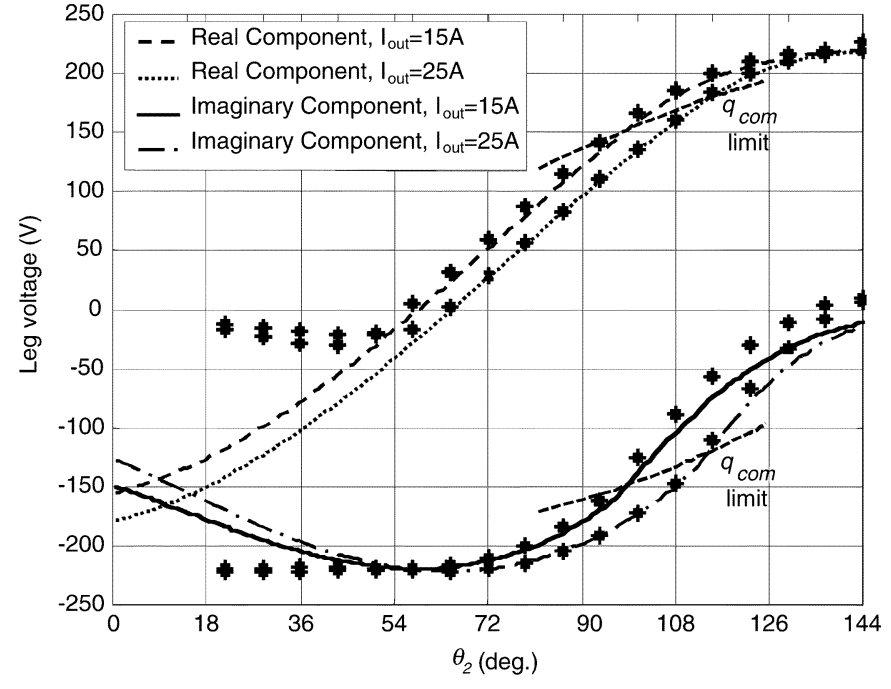

Fig. 6. Leg voltage components.

main leg voltage waveform. The resulting voltages from SPICE simulations along with those from the describing function are compared in Fig. 6. To preserve the phase information, results are analyzed by considering both the Real and Imaginary components of the leg voltage, individually. The maximum applied pulse-width is limited by the necessity for the commutation capacitors to fully charge to the dc-link rail voltage before the output current changes polarity. This is indicated in Fig. 6 by the " $q_{\text {com }}$ limit." Values of $\theta_{2}$ greater than this give rise to truncated commutation, whereby the capacitor is partly discharged by the opposing transistor turning on. The good correlation between results from the derived model (line) and those from SPICE simulations, Fig. 6, indicate that the dominant characteristics of the inverter leg are captured by the proposed model, particularly for $\theta_{2}>\pi / 3$ radians $\left(60^{\circ}\right)$ (conduction time of switch $\approx 0.8 \mu \mathrm{s}$ ); below this value diode reverse recovery dominates the turn-off characteristics. It is also of note that some discrepancy between the results occurs above the $q_{\text {com }}$ limit (especially the imaginary component) due to the assumption that a device switches-on at the instant that the current passes through zero $(\theta=0, \pi, 2 \pi$ etc). If the total switch capacitance is not fully charged at the end of half a switching cycle, a result of employing a piecewise model is that it necessarily predicts a step change in the leg voltage. This cannot occur in practice since it implies a finite amount of charge must be transferred to the commutation capacitance in zero time. The domain of applicability of the model is therefore bounded by the assumption that complete charging of the commutation capacitance can occur during half a switching cycle. In reality, circuit operation outside this domain results in large transient current flows through the switching devices, leading to a significant increase in both switching device loss and electromagnetic noise.

\section{MODEL REDUCTION}

Although the proposed model incorporates the dominant characteristic of switching behavior, the complexity can be reduced by considering the impact of each parameter $\left(C_{\text {comm }}, V_{t}, R_{\text {on }}, V_{d}, R_{d}\right.$ ) on the FHA equivalent output voltage of the bridge leg $\left(\mathrm{V}_{\mathrm{Leg}}\right)$. Applying a sinusoidal current 

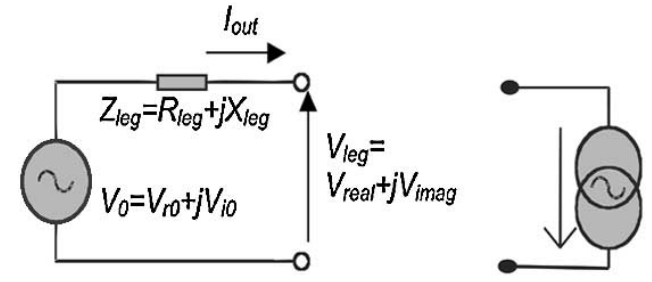

Fig. 7. Thévenin equivalent source.

sink to the output of the piecewise description of the bridge leg, resulting in an output voltage $V_{\text {Leg }}$, allows the impact of variations in the circuit parameters to be determined by considering a Thévenin equivalent circuit representation (Fig. 7).

As the proposed model of the bridge leg is only valid for a defined range of output currents viz. those allowing correct commutation of the bridge leg, two output current levels selected from small perturbations about a nominal operating point, are used to find the Thévenin components, $Z_{\text {leg }}$ and $V_{0}$, for each perturbation of either $C_{\text {comm }}, V_{t}, R_{\text {on }}, V_{d}, R_{d}$.

Each component is selected individually for analysis, and its value is halved; a "new" Thévenin equivalent circuit then being obtained for each case. Sensitivity to variations in each component is assessed by repeating the process for a range of pulse widths $\left(\theta_{1}\right)$, and calculating the RMS variation of the Thévenin model components from those of the original. The results of these analyzes are summarized in Fig. 8, from which it can be seen the components that have the greatest influence on output voltage are $C_{\text {comm }}, V_{t}$ and $R_{\text {on }}$. Consequently, the original piecewise time-domain model [(3)-(9)] can be simplified to (10) by only including the influence of these elements. In particular, the output is assumed to begin at exactly the dc-link voltage while the diode is forward biased $\left(\theta=0 \rightarrow \theta_{d}\right)$, and then be subject to a voltage drop due to the body resistance of the MOSFET until the end of the MOSFET turn-off period $\left(\theta=\theta_{d} \rightarrow \theta_{2}\right)$. Although the actual turn-off ramp is not now directly considered, the time period of the ramp is, since the commutation capacitor charging period is very sensitive to the starting time. The commutation capacitor charging profile is also dependent on the starting voltage, so the portion of the leg-voltage due to commutation begins at an angle $\theta_{2}$, and voltage, $V_{\mathrm{dc}}-V_{t}$. This implies a discontinuity in the characteristic of $\approx-V_{t}$ at $\theta_{2}$. After the commutation event (at $\theta_{3}$ ), the voltage is clamped to $0 \mathrm{~V}$. The second half of the operation sequence then commences analogously as (10), shown at the bottom of the page. (a)

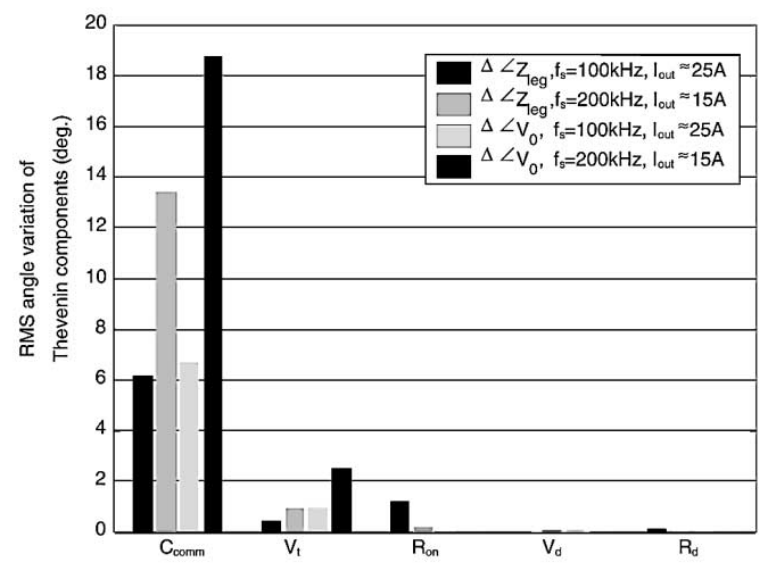

(b)

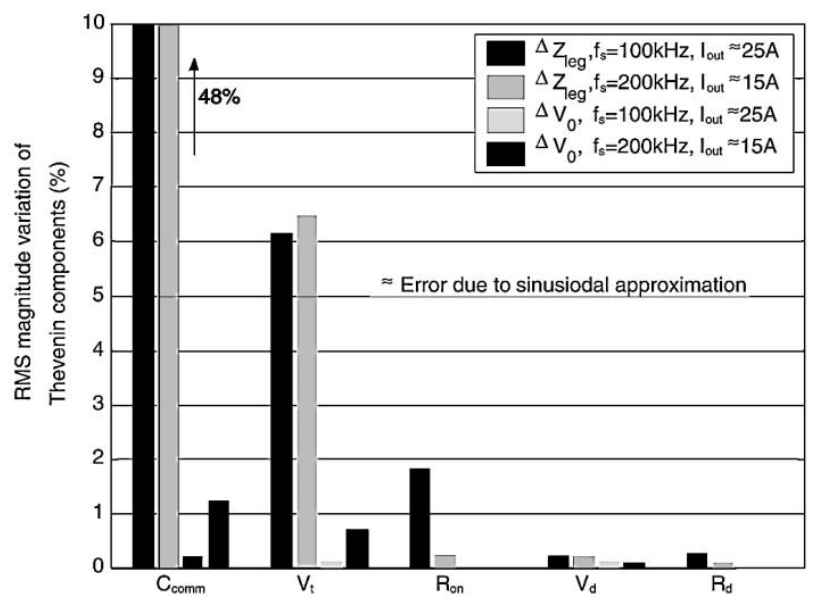

Fig. 8. Effect of different elements of the time-domain description on the Thévenin equivalent circuit (a) angle and (b) magnitude.

\section{DESCRIBING FUNCTION OF INVERTER LEG}

A describing function to model the inverter leg is obtained from a FHA of (10). This is most conveniently found from the first harmonic of the Fourier series of the leg voltage described in the "angle-domain," Since the relative phase shift of the output voltage is important, the complex form of the output voltage vector is retained $\left(V_{\mathrm{Leg}}(\mathrm{FMA})=V_{\text {real }}+j V_{\text {imag }}\right)$, resulting in the describing function being comprised of both Real and Imaginary components (11), (12). To simplify notation, $i_{\text {out }}$ is also divided into constituent components $I_{\sin }$ and $I_{\cos }$, where $I_{\sin }$ is in phase with $V_{\text {real }}$, and $I_{\cos }$ correspond-

$$
v_{\mathrm{Leg}}(\theta)= \begin{cases}V_{\mathrm{dc}}, & \theta=0 \ldots \theta_{d} \\ V_{\mathrm{dc}}-I_{\mathrm{out}} R_{\mathrm{on}} \sin \left(\theta-\theta_{d}\right), & \theta=\theta_{d} \ldots \theta_{2} \\ \frac{I_{\mathrm{out}}}{2 \pi f_{s} C_{\mathrm{comm}}} \cos \left(\theta-\theta_{d}\right)+V_{\mathrm{dc}}-V_{t}-\frac{I_{\mathrm{out}}}{2 \pi f_{s} C_{\mathrm{comm}}} \cos \left(\theta_{d}-\theta_{2}\right), & \theta=\theta_{2} \ldots \theta_{3} \\ 0, & \theta=\theta_{3} \ldots \pi+\theta_{d} \\ -I_{\text {out }} R_{\mathrm{on}} \sin \left(\theta-\theta_{d}\right), & \theta=\pi+\theta_{d} \ldots \pi+\theta_{2} \\ \frac{I_{\text {out }}}{2 \pi f_{s} C_{\mathrm{comm}}} \cos \left(\theta-\theta_{d}\right)+V_{t}-\frac{I_{\text {out }}}{2 \pi f_{s} C_{\mathrm{comm}}} \cos \left(\theta_{d}-\theta_{2}+\pi\right), & \theta=\pi+\theta_{2} \ldots \pi+\theta_{3} \\ V_{\mathrm{dc}}, & \theta=\pi+\theta_{3} \ldots 2 \pi\end{cases}
$$


ingly leading by $\pi / 2$ radians; thus $I_{\text {out }}=\sqrt{I_{\text {sin }}^{2}+I_{\text {cos }}^{2}}$ and $\tan \left(\theta_{d}\right)=\left(I_{\sin }\right) /\left(I_{\cos }\right)$

$$
\begin{aligned}
V_{\text {real }}= & \left\{-\frac{2 V_{\mathrm{DC}}}{\pi} \cos \left(\theta_{3}\right)+\frac{R_{\mathrm{on}}}{2 \pi}\left(\left(\sin \left(2 \theta_{2}\right)-2 \theta_{2}\right) I_{\text {sin }}\right.\right. \\
& \left.+\left(\cos \left(2 \theta_{2}\right)-1\right) I_{\mathrm{cos}}\right) \\
& +\frac{2 V_{t}}{\pi}\left(\cos \left(\theta_{3}\right)-\cos \left(\theta_{2}\right)\right) \\
& +\frac{I_{\mathrm{sin}}}{4 \pi^{2} f_{s} C_{\mathrm{comm}}}\left(4 \cos \left(\theta_{2}\right) \cos \left(\theta_{3}\right)\right. \\
& \left.-\cos \left(2 \theta_{3}\right)-\cos \left(2 \theta_{2}\right)-2\right) \\
& +\frac{I_{\mathrm{cos}}}{4 \pi^{2} f_{s} C_{\mathrm{comm}}}\left(2 \theta_{2}-2 \theta_{3}-4 \sin \left(\theta_{2}\right) \cos \left(\theta_{3}\right)\right. \\
& \left.\left.+\sin \left(2 \theta_{2}\right)+\sin \left(2 \theta_{3}\right)\right)\right\} \\
V_{\text {imag }}= & \left\{\frac{2 V_{\mathrm{DC}}}{\pi} \sin \left(\theta_{3}\right)+\frac{R_{\mathrm{on}}}{2 \pi}\left(\left(\cos \left(2 \theta_{2}\right)-1\right) I_{\mathrm{sin}}\right.\right. \\
& \left.+\left(-\sin \left(2 \theta_{2}\right)-2 \theta_{2}\right) I_{\mathrm{cos}}\right) \\
& +\frac{2 V_{t}}{\pi}\left(\sin \left(\theta_{2}\right)-\sin \left(\theta_{3}\right)\right) \\
& +\frac{I_{\mathrm{sin}}}{4 \pi^{2} f_{s} C_{\mathrm{comm}}}\left(-2 \theta_{2}+2 \theta_{3}+\sin \left(2 \theta_{2}\right)\right. \\
& \left.+\sin \left(2 \theta_{3}\right)-4 \cos \left(\theta_{2}\right) \sin \left(\theta_{3}\right)\right) \\
& +\frac{I_{\mathrm{cos}}}{4 \pi^{2} f_{s} C_{\mathrm{comm}}}\left(4 \sin \left(\theta_{2}\right) \sin \left(\theta_{3}\right)\right. \\
& \left.\left.+\cos \left(2 \theta_{2}\right)+\cos \left(2 \theta_{3}\right)-2\right)\right\}
\end{aligned}
$$

The switching behavior of a single inverter leg can now be generalized to model both legs of the inverter H-bridge; the output voltage then being between the mid-point node of each leg.

To identify each set of equations with the appropriate leg, $\alpha_{\{1,2,3 \ldots\}}, C_{\text {comm(PWM) }}^{\prime}$ and $\beta_{\{1,2,3 \ldots\}}, C_{\text {comm(LOAD) }}^{\prime}$ are employed, respectively, for describing the characteristics of the "PWM-commutated leg" and the "load commutated leg," where the angles $\alpha_{\{1,2,3 \ldots\}}$ replace the general angles $\theta_{\{1,2,3 \ldots\}}$ to mark the various switching events in the PWM-commutated leg, and the angles $\beta_{\{1,2,3 \ldots\}}$ replace the general angles $\theta_{\{1,2,3 \ldots\}}$ to mark the various switching events in the load-commutated leg. The output voltage is found by effectively subtracting the voltage of the "load-commutated leg" from that of the "PWM leg." Now, since the upper switch in the PWM leg is turned on $\pi$ radians out of phase with the load-commutated leg, and the output current similarly reversed, the voltage at the PWM leg must be derived with a $\pi$ radians phase-shift. Therefore, the output voltage is actually found by adding the voltage contribution of each leg

$$
\begin{aligned}
V_{\text {out }}= & \left\{-\frac{2 V_{\mathrm{DC}}}{\pi}\left(\cos \left(\alpha_{3}\right)+\cos \left(\beta_{3}\right)\right)\right. \\
& +\frac{R_{\mathrm{on}}}{2 \pi}\left(\left(\sin \left(2 \alpha_{2}\right)+\sin \left(2 \beta_{2}\right)-2 \alpha_{2}-2 \beta_{2}\right) I_{\mathrm{sin}}\right. \\
& \left.+\left(\cos \left(2 \alpha_{2}\right)+\cos \left(2 \beta_{2}\right)-2\right) I_{\mathrm{cos}}\right) \\
& +\frac{2 V_{t}}{\pi}\left(\cos \left(\alpha_{3}\right)+\cos \left(\beta_{3}\right)-\cos \left(\alpha_{2}\right)-\cos \left(\beta_{2}\right)\right) \\
& +\frac{I_{\mathrm{sin}}}{4 \pi^{2} f_{s} C_{\mathrm{comm}(\mathrm{PWM})}^{\prime}}\left(4 \cos \left(\alpha_{2}\right) \cos \left(\alpha_{3}\right)\right. \\
& \left.-\cos \left(2 \alpha_{3}\right)-\cos \left(2 \alpha_{2}\right)-2\right)
\end{aligned}
$$

$$
\begin{aligned}
& +\frac{I_{\mathrm{cos}}}{4 \pi^{2} f_{s} C_{\mathrm{comm}(\mathrm{PWM})}^{\prime}}\left(2 \alpha_{2}-2 \alpha_{3}\right. \\
& \left.-4 \sin \left(\alpha_{2}\right) \cos \left(\alpha_{3}\right)+\sin \left(2 \alpha_{2}\right)+\sin \left(2 \alpha_{3}\right)\right) \\
& +\frac{I_{\text {sin }}}{4 \pi^{2} f_{s} C_{\text {comm (Load) }}^{\prime}}\left(4 \cos \left(\beta_{2}\right) \cos \left(\beta_{3}\right)\right. \\
& \left.-\cos \left(2 \beta_{3}\right)-\cos \left(2 \beta_{2}\right)-2\right) \\
& +\frac{I_{\text {cos }}}{4 \pi^{2} f_{s} C_{\text {comm(Load) }}^{\prime}}\left(2 \beta_{2}-2 \beta_{3}\right. \\
& \left.\left.-4 \sin \left(\beta_{2}\right) \cos \left(\beta_{3}\right)+\sin \left(2 \beta_{2}\right)+\sin \left(2 \beta_{3}\right)\right)\right\} \\
& +j\left\{\frac{2 V_{\mathrm{DC}}}{\pi}\left(\sin \left(\alpha_{3}\right)+\sin \left(\beta_{3}\right)\right)\right. \\
& +\frac{R_{\mathrm{on}}}{2 \pi}\left(\left(\cos \left(2 \alpha_{2}\right)+\cos \left(2 \beta_{2}\right)-2\right) I_{\text {sin }}\right. \\
& \left.-\left(\sin \left(2 \alpha_{2}\right)+\sin \left(2 \beta_{2}\right)+2 \alpha_{2}+2 \beta_{2}\right) I_{\cos }\right) \\
& +\frac{2 V_{t}}{\pi}\left(\sin \left(\alpha_{2}\right)-\sin \left(\alpha_{3}\right)+\sin \left(\beta_{2}\right)-\sin \left(\beta_{3}\right)\right) \\
& +\frac{I_{\text {sin }}}{4 \pi^{2} f_{s} C_{\text {comm }(\mathrm{PWM})}^{\prime}}\left(-2 \alpha_{2}+2 \alpha_{3}+\sin \left(2 \alpha_{2}\right)\right. \\
& \left.+\sin \left(2 \alpha_{3}\right)-4 \cos \left(\alpha_{2}\right) \sin \left(\alpha_{3}\right)\right) \\
& +\frac{I_{\text {cos }}}{4 \pi^{2} f_{s} C_{\text {comm }(\mathrm{PWM})}^{\prime}}\left(4 \sin \left(\alpha_{2}\right) \sin \left(\alpha_{3}\right)\right. \\
& \left.+\cos \left(2 \alpha_{2}\right)+\cos \left(2 \alpha_{3}\right)-2\right) \\
& +\frac{I_{\text {sin }}}{4 \pi^{2} f_{s} C_{\text {comm(Load) }}^{\prime}}\left(-2 \beta_{2}+2 \beta_{3}+\sin \left(2 \beta_{2}\right)\right. \\
& \left.+\sin \left(2 \beta_{3}\right)-4 \cos \left(\beta_{2}\right) \sin \left(\beta_{3}\right)\right) \\
& +\frac{I_{\text {cos }}}{4 \pi^{2} f_{s} C_{\text {comm(Load) }}^{\prime}}\left(4 \sin \left(\beta_{2}\right) \sin \left(\beta_{3}\right)\right. \\
& \left.\left.+\cos \left(2 \beta_{2}\right)+\cos \left(2 \beta_{3}\right)-2\right)\right\} \text {. }
\end{aligned}
$$

It should be noted that $C_{\text {comm(Load) }}^{\prime}, C_{\text {comm(PWM) }}^{\prime}$ are "equivalent capacitances" that include the nonlinear parasitic output capacitance of the switching device. A degree of verification of the model can be seen from Fig. 9, where, by fixing $\alpha_{1}, V_{\mathrm{DC}}$ and $I_{\text {out }}$, and varying $\beta_{1}$, a good correlation between SPICE and the derived results is evident for values of device on-time, $T_{\text {on }}$, greater than the diode recovery time of $0.8 \mu \mathrm{s}$.

\section{A. Output Voltage Range Versus Frequency}

As the operating frequency is increased, the required commutation capacitor charge time leads to a relative increase in the switching time of the leg with respect to the switching period. This acts to increase the output voltage for low values of output pulse width, controlled by $\beta_{1}$ ( $\alpha_{1}$ being fixed), while, conversely, acting to decrease the output voltage for high pulse widths ( $\beta_{3}$ approaching $\pi$ radians). It is therefore apparent that the commutation capacitor charging-time constrains the range of $\beta_{1}$, hence limiting the time that the output voltage can be clamped to the supply.

\section{B. Input Current}

The average current drawn by the inverter is the product of the total charge drawn from the source over a switching cycle, and the switching frequency. From Figs. 1 and 10 (which shows one 

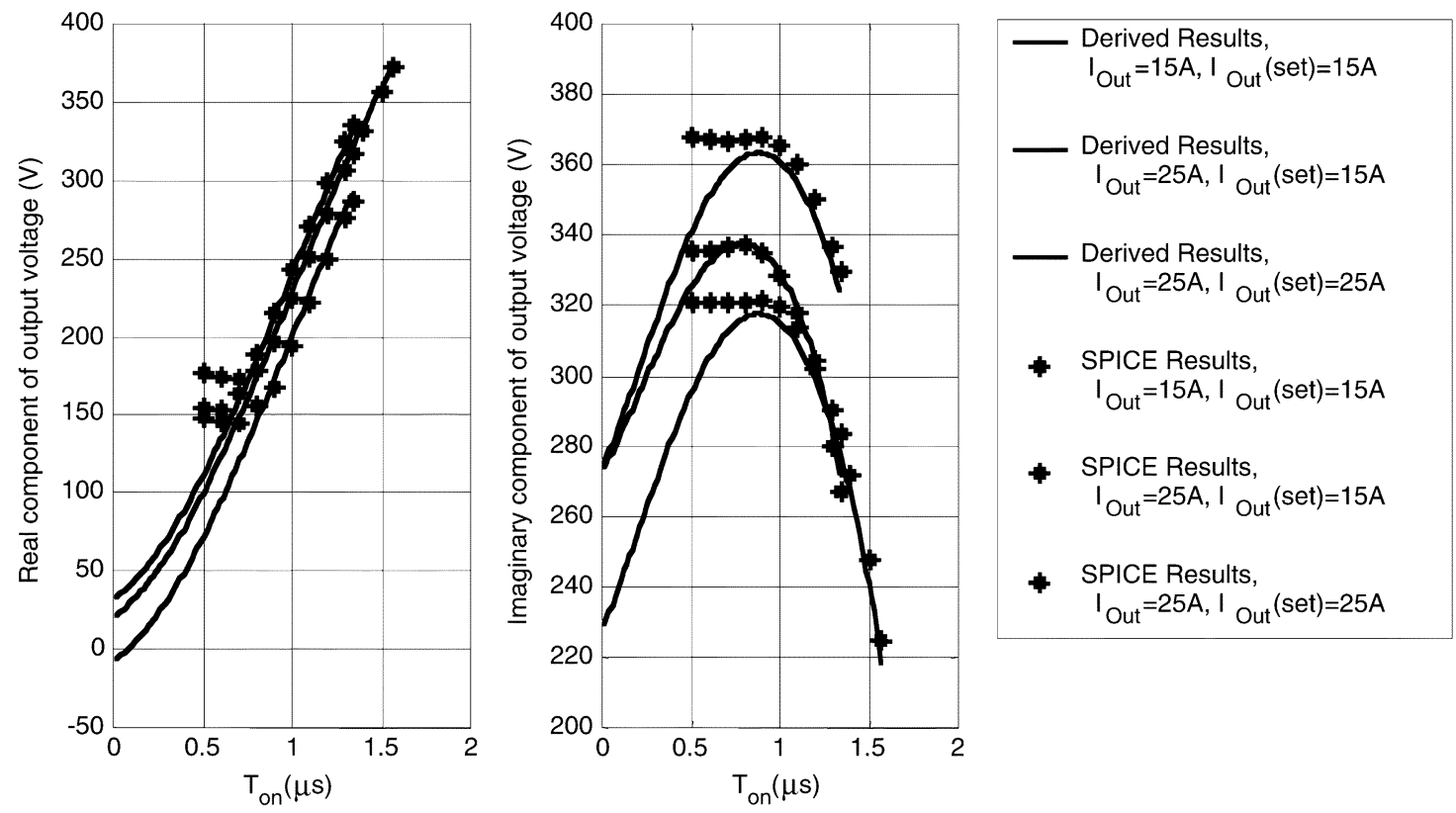

Fig. 9. Total output voltage from the inverter.

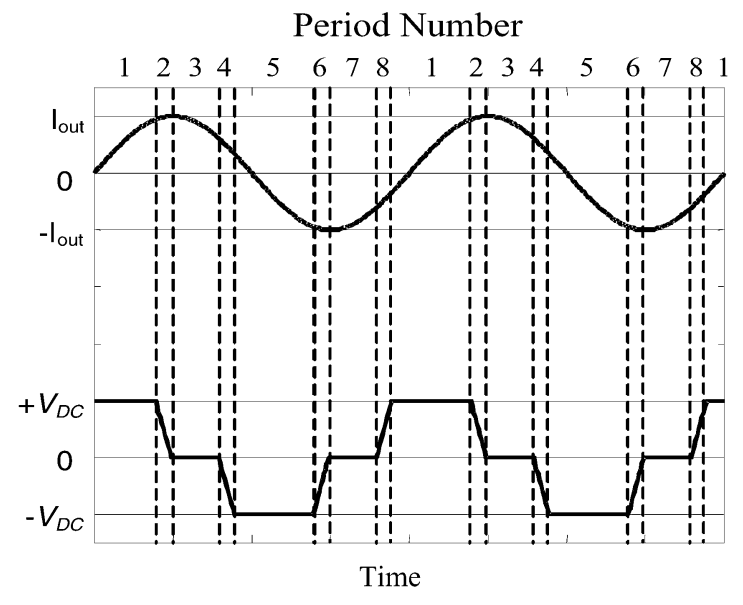

Fig. 10. Definition of Periods 1 to 8.

cycle of the inverter output voltage and current subdivided into time-domain piece-wise components), an analytical function for the averaged supply input current can be obtained by successively considering each of the eight steady-state operating periods.

1) Period 1: $\left\{\left(\alpha_{3}-\pi\right)\right.$ to $\left.\beta_{2}\right\}$ : During Period 1 the load is connected across the supply by $\mathrm{D}_{1}$ and $\mathrm{D}_{4}$, and subsequently by the operation of $\mathrm{SW}_{1}$ and $\mathrm{SW}_{4}$. The charge flowing from the supply for this period can be seen to be a function of the output current. Assuming the output current is sinusoidal with a variable phase-shift such that the diodes do not become reverse biased before their anti-parallel transistors turn on, the charge can be found by integration

$$
\begin{aligned}
q_{1}= & \frac{1}{2 \pi f_{s}} \int_{\alpha_{3}-\pi}^{\beta_{2}} i_{\text {out }}(\theta) d \theta \\
= & \frac{I_{\cos }}{2 \pi f_{s}}\left[\sin \left(\alpha_{3}\right)+\sin \left(\beta_{2}\right)\right] \\
& -\frac{I_{\text {sin }}}{2 \pi f_{s}}\left[\cos \left(\alpha_{3}\right)+\cos \left(\beta_{2}\right)\right] .
\end{aligned}
$$

Although the solution is dependent on output current (amplitude and phase) and commutation angles, it is not explicitly dependent on supply voltage. However, implicitly, the output current is a function of the (complex) output voltage, which, in turn, is a function of the supply voltage. Moreover, $\alpha_{3}$ is a function of the supply voltage and the output current.

2) Period 2: $\left\{\beta_{2}\right.$ to $\left.\beta_{3}\right\}$ : Here, the load current is apportioned to discharging $\mathrm{C}_{2}$ and charging $\mathrm{C}_{4}$ (from the supply), the magnitude of each being determined by the ratio of capacitances $C_{2}: C_{4}$. Since the parasitic capacitances across the switching devices vary as a function of applied voltage, the instantaneous supply current varies over the commutation period. The charge flowing from the dc link is therefore equal to that required to charge $\mathrm{C}_{4}$ (plus the parasitic capacitance across the source-drain terminals of $\mathrm{SW}_{4}$ ) from $0 \mathrm{~V}$ to $V_{\mathrm{DC}}(15)$. The function relating the charge stored in the nonlinear parasitic capacitor across the MOSFET, to the dc link voltage, $F_{c}\left(V_{\mathrm{dc}}\right)$, is found by integrating the MOSFET output capacitance, $C_{\mathrm{oss}}$, as a function of $v_{\mathrm{ds}}$

$$
q_{2}=C_{4} V_{\mathrm{DC}}+F_{c}\left(V_{\mathrm{DC}}\right)
$$

Equation (15) is also approximately correct given the case of truncated commutation, since, if $\mathrm{C}_{2}$ is not completely discharged at the end of the cycle, $\mathrm{SW}_{2}$ will turn-on and charge $\mathrm{C}_{4}$ from the dc link, while rapidly discharging $\mathrm{C}_{2}$. The energy stored in $\mathrm{C}_{2}$ will then dissipate in $\mathrm{SW}_{2}$, increasing switching loss.

3) Period 3: $\left\{\beta_{3}\right.$ to $\left.\alpha_{2}\right\}$ : The output current circulates through $\mathrm{SW}_{1}$ and $\mathrm{D}_{2}$, and hence, no current is drawn from the supply

$$
q_{3}=0
$$

4) Period 4: $\left\{\alpha_{2}\right.$ to $\left.\alpha_{3}\right\}$ : The load current is divided into a portion circulating via $\mathrm{C}_{1}$, and the other from the load, through $\mathrm{C}_{3}$ and into the supply. The current to the supply is of opposite 
polarity to that discussed for Period 2: therefore energy is returned. The charge flowing from the supply to the H-Bridge is therefore that required to discharge $\mathrm{C}_{3}$ and the parasitic capacitance across $\mathrm{SW}_{3}$

$$
q_{4}=-C_{3} V_{\mathrm{DC}}-F_{c}\left(V_{\mathrm{DC}}\right) .
$$

5) Period 5: $\left\{\alpha_{3}\right.$ to $\left.\left(\pi+\beta_{2}\right)\right\}$ : Initially, current flows from the load, via $\mathrm{D}_{2}$ and $\mathrm{D}_{3}$, to the supply; on reversal it flows from the supply, through $\mathrm{SW}_{2}$ and $\mathrm{SW}_{3}$ to the load, (similar to Period 1)

$$
q_{5}=\frac{1}{2 \pi f_{s}} \int_{\alpha_{3}}^{\beta_{2}+\pi}-i_{\text {out }}(\theta) d \theta .
$$

The sign change is due to the voltage across the load being reversed. Considering the sign change as a phase shift of $\pi$ radians, (18) simplifies to (19), which is equivalent to $q_{1}$

$$
\begin{aligned}
q_{5}=\frac{I_{\cos }}{2 \pi f_{s}}\left[\sin \left(\alpha_{3}\right)+\right. & \left.\sin \left(\beta_{2}\right)\right] \\
& -\frac{I_{\sin }}{2 \pi f_{s}}\left[\cos \left(\alpha_{3}\right)+\cos \left(\beta_{2}\right)\right]=q_{1} .
\end{aligned}
$$

6) Period 6: $\left\{\left(\pi+\beta_{2}\right)\right.$ to $\left.\left(\pi+\beta_{3}\right)\right\}$ : Here, current is drawn from the supply in charging $\mathrm{C}_{2}$ via the load. If $C_{2}$ is equal to $C_{4}$, then $q_{6}$ is equal to $q_{2}$

$$
q_{6}=C_{2} V_{\mathrm{DC}}+F_{c}\left(V_{\mathrm{DC}}\right) .
$$

7) Periods $7 \&$ 8: During Period 7, the load current circulates through $\mathrm{SW}_{3}$ and $\mathrm{D}_{4}$ (similar to Period 3) and the charge from the supply will be zero

$$
q_{7}=0 .
$$

During Period 8, $\mathrm{SW}_{3}$ commutates, with some of the output current flowing through $\mathrm{C}_{3}$ and $\mathrm{D}_{4}$, the remainder flowing through $\mathrm{C}_{1}, \mathrm{D}_{4}$ to the supply. Charge flowing into the supply is therefore equal to the requirements to charge $\mathrm{C}_{1}$ from $0 \mathrm{~V}$ to $+V_{\mathrm{DC}}$ [see (22)]. If $C_{1}$ equals $C_{3}$, then $q_{8}$ is equal to $q_{4}$

$$
q_{8}=-C_{1} V_{\mathrm{DC}}-F_{c}\left(V_{\mathrm{DC}}\right) .
$$

a) Average Input Current: The average input current is given by the product of the sum of $q_{1} \rightarrow q_{8}$, and the switching frequency. It is notable that terms relating to the charge stored in the parasitic capacitance of the MOSFETs cancel, leaving

$$
\begin{aligned}
I_{\mathrm{dc}}=\frac{I_{\mathrm{cos}}}{\pi}\left[\sin \left(\alpha_{3}\right)+\right. & \left.\sin \left(\beta_{2}\right)\right]-\frac{I_{\mathrm{sin}}}{\pi}\left[\cos \left(\alpha_{3}\right)+\cos \left(\beta_{2}\right)\right] \\
& +\left(C_{2}+C_{4}-C_{1}-C_{3}\right) V_{\mathrm{DC}} f_{s} .
\end{aligned}
$$

A comparison of results of $I_{\mathrm{dc}}$ from (23), with those from SPICE simulations, is given in Fig. 11 for a variety of $\beta_{2}$ and $I_{\text {out }}$, where a close agreement is apparent for $T_{\text {on }}>\sim 0.8 \mu \mathrm{s}$ (corresponding to the reverse recovery time of the SPICE-model diodes). The high degree of correlation remains even when the PWM leg undergoes truncated commutation (i.e., when $T_{\text {on }}$ is greater than the $q_{\mathrm{com}}$ limit), which is consistent with our analysis, which does not consider the destination of the charge during the commutation period.

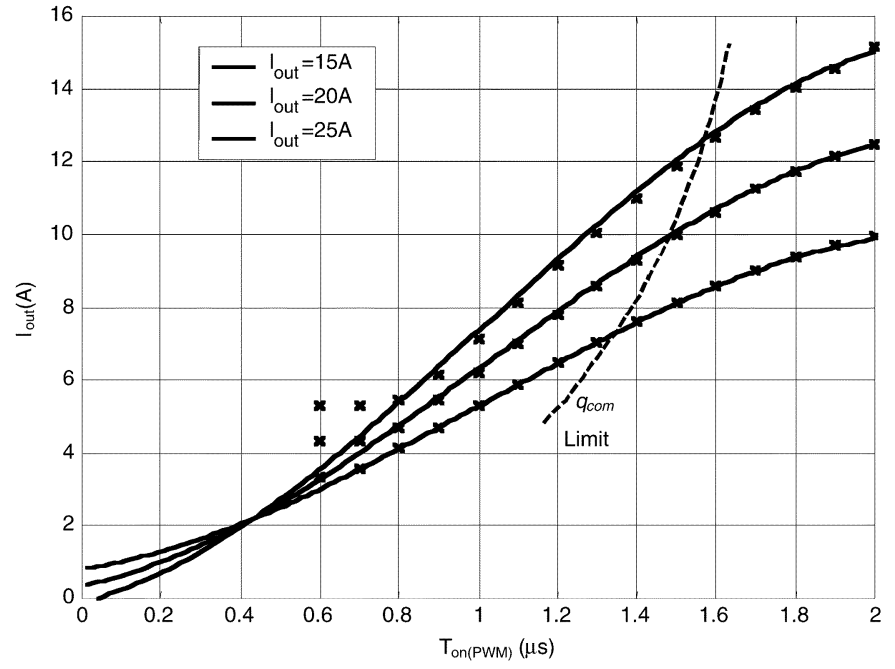

Fig. 11. Average input current as a function of PWM gate pulse-width.

(a)

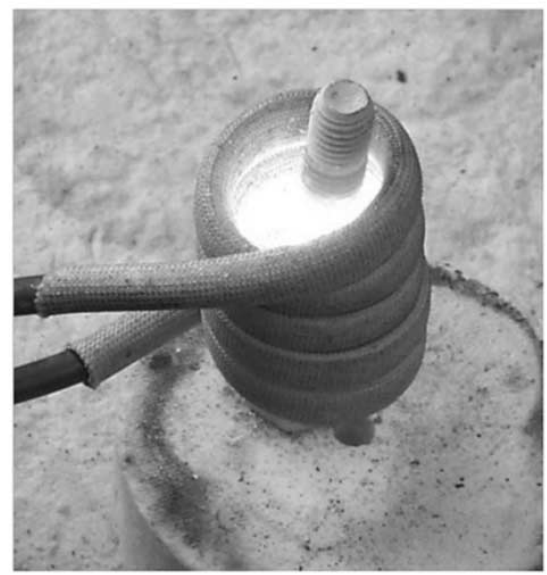

(b)

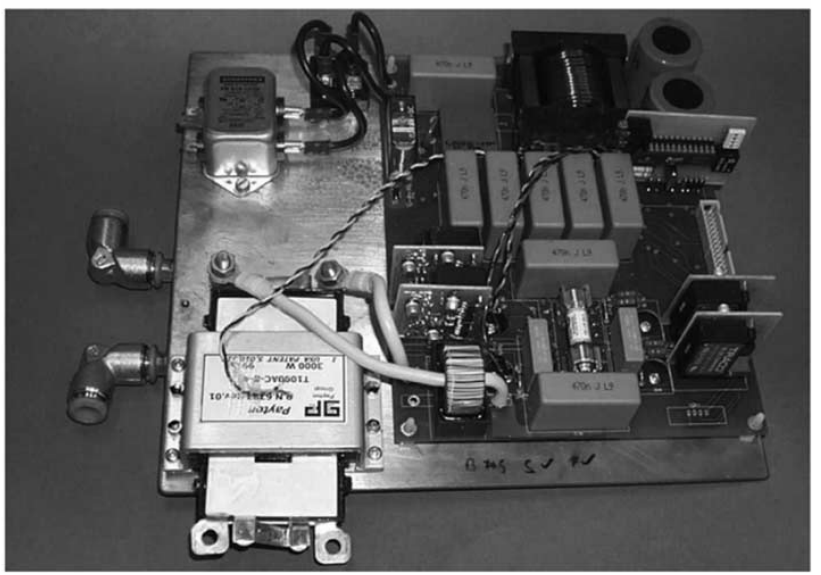

Fig. 12. Prototype induction heating system (a) application heating a bolt to $1000^{\circ} \mathrm{C}$ and (b) $2.5-\mathrm{kW}$ inverter circuit.

Experimental measurements from a prototype induction heating system, shown in Fig. 12, (whose parameters have been accurately measured) have also been obtained to verify the predictions. The equivalent circuit of the induction heating work-head, which represents the load on the bridge, is shown in Fig. 13. The load circuit is connected to the inverter, with 


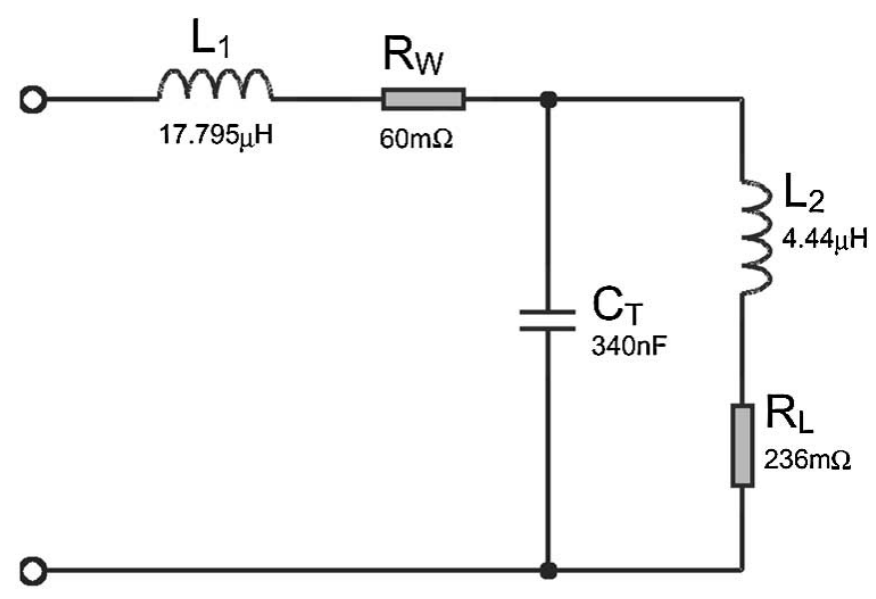

Fig. 13. Equivalent work-head circuit parameters.

TABLE I

COMPONENT VALUES USED IN THE VERIFICATION OF THE SySTEM Model

\begin{tabular}{l|l|l}
\hline Component & Description & Value \\
\hline $\mathrm{SW}_{1} \rightarrow \mathrm{SW}_{4}$ & Power switching MOSFETs & APT5101LVR \\
\hline$C_{l}, C_{3}$ & Load commutated leg commutation capacitors & $4.7 \mathrm{nF}$ \\
\hline$C_{2}, C_{4}$ & PWM commutated leg commutation capacitors & $10 \mathrm{nF}$ \\
\hline$\theta_{d}$ & Current phase set-point bias & $0^{\circ}$ \\
\hline$\alpha_{1}$ & Turn-off signal angle for the load commutated leg & $134^{\circ}$ \\
\hline
\end{tabular}

parameters shown in Table I, via a 2:1 ratio step-down transformer, and the inverter is fed from a 300-V, 9-A supply.

As $\beta_{1}$ increases, the pulse-width, and hence the output voltage from the system, increases. This is reflected in an increase in the output current from the inverter, shown as the peak output current value in Fig. 14. The input current also further increases with $\beta_{1}$ over the range of output current as the relative time available to transfer energy from the dc link to the output increases with output pulse width. However, with $\beta_{1}$ small, the effect of the commutation period is significant, therefore some excitation of the workhead circuit occurs leading to a minimum output current. Furthermore, due to the effect of the commutation capacitors, a minimum input current flows at $\beta_{1}=0$. From Fig. 14, a good correlation between the experimental data and the predicted results for the inverter is apparent [4]-[9].

\section{Model Limitations}

The presented time-domain description (10) is valid only when the switch in anti-parallel with the conducting diode at the end of the cycle, is turned-on before the current passes through zero (normal operation, $\theta_{d}$ positive). In other cases, the output current transfers from the diode to the commutation capacitors, and the modeling of an additional period is required between $\theta_{d}+\pi$ to $\pi$. During this period, the voltage at the centre of the

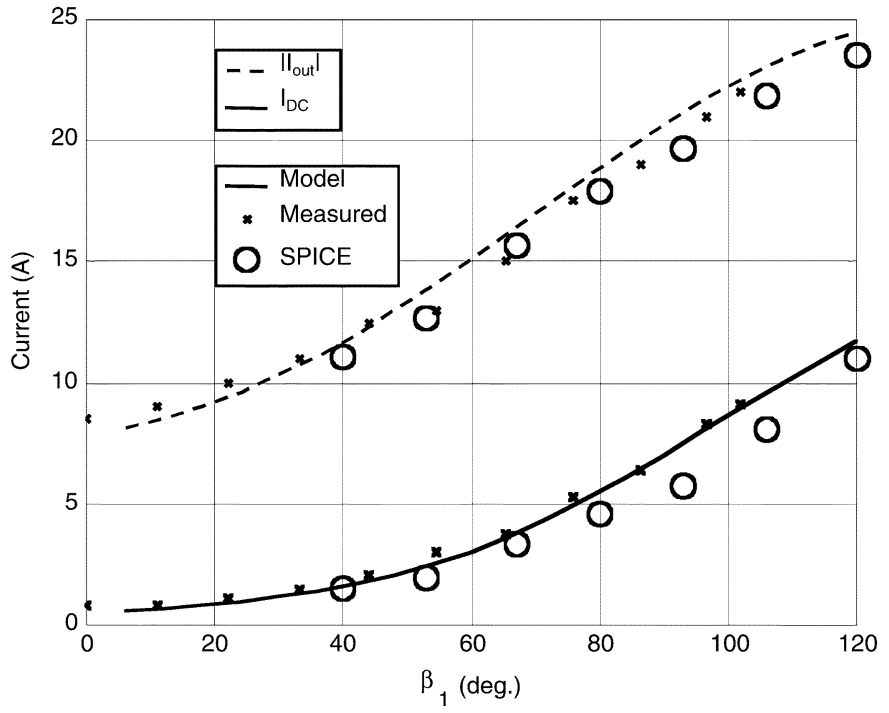

Fig. 14. Output- and input-current as a function of $\beta_{1}$, from the experimental system under test, a full system SPICE model, and the SIMULINK system model incorporating the proposed describing function.

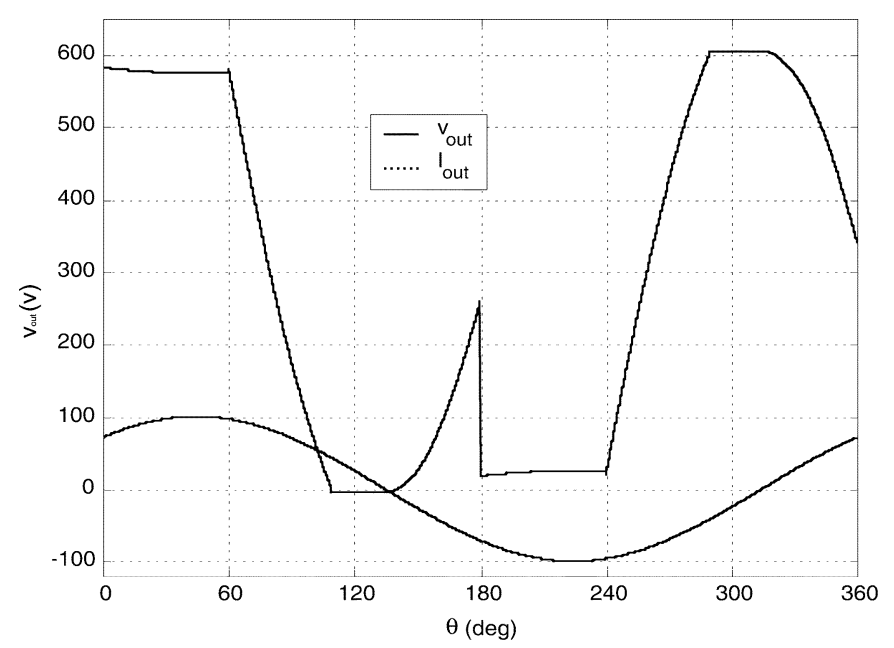

Fig. 15. Terminated commutation.

leg increases (see Fig. 15) until the switching device turns on. At the instant of turn-on, the partially charged commutation capacitor will be shorted, resulting in a large current spike (and incurring high loss). To accommodate this operating condition, (10) is modified to (24), shown at the bottom of the next page. If the output current further advances beyond the point where $\theta_{d}+\pi=\theta_{3}$, the leg voltage cannot reach the supply rail before the output current reverses, making the $d v_{\text {out }} / d t$ reverse, as shown in Fig. 16. The commutation period then terminates at the turn-on of the transistor, again incurring high loss and high $d v_{\text {out }} / d t$. Beyond this point, the calculation of $\theta_{3}$ from (7) can provide complex results, and is no longer valid. However, $\theta_{3}$ still depicts the end of the commutation cycle and, for this mode of operation, $\theta_{3}=\pi$ radians. The leg voltage is therefore 


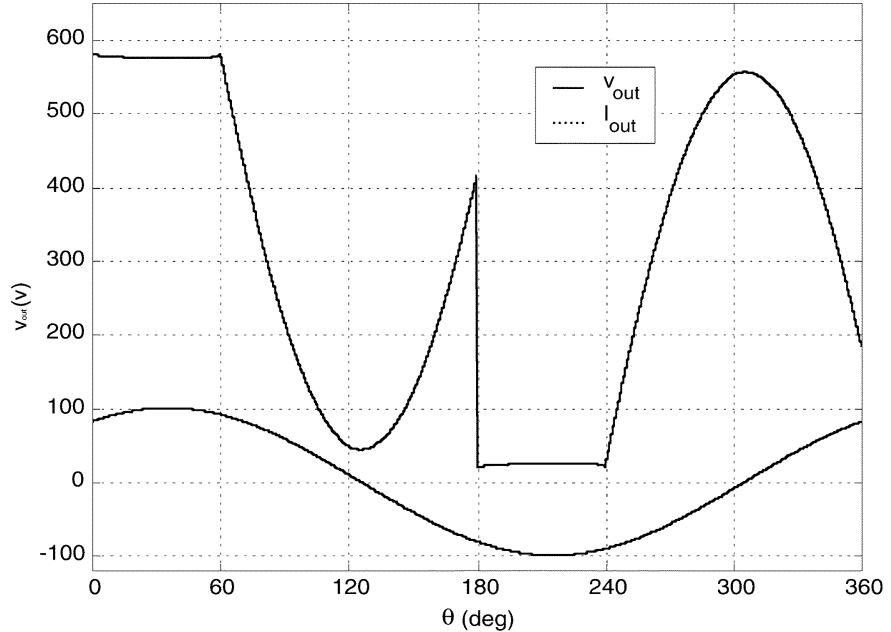

Fig. 16. $d V_{\text {out }} / d t$ reversal before leg voltage reaches $0 \mathrm{~V}$.

very similar to the case for terminated commutation (Fig. 15) as (25), shown at the bottom of the page.
If $\theta_{d}$ is advanced still further, the leg voltage, after initially reducing at device turn-off, rises to the supply voltage, whereupon the anti-parallel diode across the switch that has just turned off becomes forward biased and supports the output current. At $\theta=\pi$ radians, the lower switch turns on, and supports the output current. During turn-on, it dissipates all of the energy in the commutation capacitors and has to accommodate the diode reverse recovery; thus incurring high loss. The angle at which the diode begins to conduct, $\theta_{4}=2 \pi+2 \theta_{d}-\theta_{2}$, is obtained by exploiting symmetry of the charging waveform, see Fig. 17. $\theta_{4}$ is thus defined as the angle at which the voltage across the capacitors reach $V_{\mathrm{DC}}-V_{t}$. In reality, the voltage will rise to $V_{\mathrm{DC}}+V_{d}$, and, the exploitation of symmetry in this manner introduces an error into the calculation. However, since the resulting $d v_{\text {out }} / d t$ at $\theta_{4}$ is high, and the voltage error $\left(=V_{\text {diode }}+V_{t}\right)$ is low, the timing error introduced is small; except in cases when $\theta_{d}$ approaches $-\pi / 2$ or $V_{\mathrm{DC}}$ is very low. This mode occurs if $\theta_{d} \leq\left(\theta_{2} / 2\right)-(\pi / 2)$ (does not account for the effects of $V_{t}$ and $V_{\text {diode }}$ ) as (26), shown at the very bottom of the page.

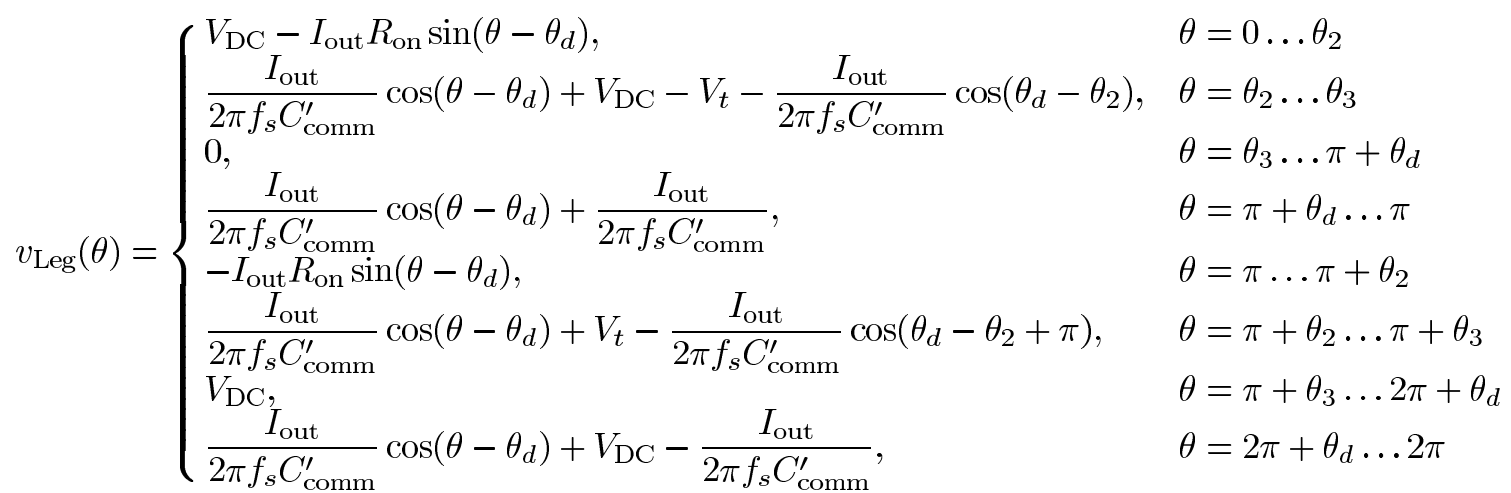$$
v_{\mathrm{Leg}}(\theta)= \begin{cases}V_{\mathrm{DC}}-I_{\mathrm{out}} R_{\mathrm{on}} \sin \left(\theta-\theta_{d}\right), & \theta=0 \ldots \theta_{2} \\ \frac{I_{\mathrm{out}}}{2 \pi f_{s} C_{\mathrm{comm}}^{\prime}} \cos \left(\theta-\theta_{d}\right)+V_{\mathrm{DC}}-V_{t}-\frac{I_{\mathrm{out}}}{2 \pi f_{s} C_{\mathrm{comm}}^{\prime}} \cos \left(\theta_{d}-\theta_{2}\right), & \theta=\theta_{2} \ldots \pi \\ -I_{\mathrm{out}} R_{\mathrm{on}} \sin \left(\theta-\theta_{d}\right), & \theta=\pi \ldots \pi+\theta_{2} \\ \frac{I_{\mathrm{out}}}{2 \pi f_{s} C_{\mathrm{comm}}^{\prime}} \cos \left(\theta-\theta_{d}\right)+V_{t}-\frac{I_{\mathrm{out}}}{2 \pi f_{s} C_{\mathrm{comm}}} \cos \left(\theta_{d}-\theta_{2}+\pi\right), & \theta=\pi+\theta_{2} \ldots 2 \pi\end{cases}
$$

$$
v_{\mathrm{Leg}}(\theta)= \begin{cases}V_{\mathrm{dc}}-I_{\text {out }} R_{\mathrm{on}} \sin \left(\theta-\theta_{d}\right), & \theta=0 \ldots \theta_{2} \\ \frac{I_{\mathrm{out}}}{2 \pi f_{s} C_{\mathrm{comm}}^{\prime}} \cos \left(\theta-\theta_{d}\right)+V_{\mathrm{dc}}-V_{t}-\frac{I_{\text {out }}}{2 \pi f_{s} C_{\mathrm{comm}}^{\prime}} \cos \left(\theta_{d}-\theta_{2}\right), & \theta=\theta_{2} \ldots \theta_{4} \\ V_{\mathrm{dc}}, & \theta=\theta_{4} \ldots \pi \\ -I_{\text {out }} R_{\mathrm{on}} \sin \left(\theta-\theta_{d}\right), & \theta=\pi \ldots \pi+\theta_{2} \\ \frac{I_{\text {out }}}{2 \pi f_{s} C_{\mathrm{comm}}^{\prime}} \cos \left(\theta-\theta_{d}\right)+V_{t}-\frac{I_{\mathrm{out}}}{2 \pi f_{s} C_{\mathrm{comm}}^{\prime}} \cos \left(\theta_{d}-\theta_{2}+\pi\right), & \theta=\pi+\theta_{2} \ldots \pi+\theta_{4} \\ 0, & \theta=\pi+\theta_{4} \ldots 2 \pi\end{cases}
$$




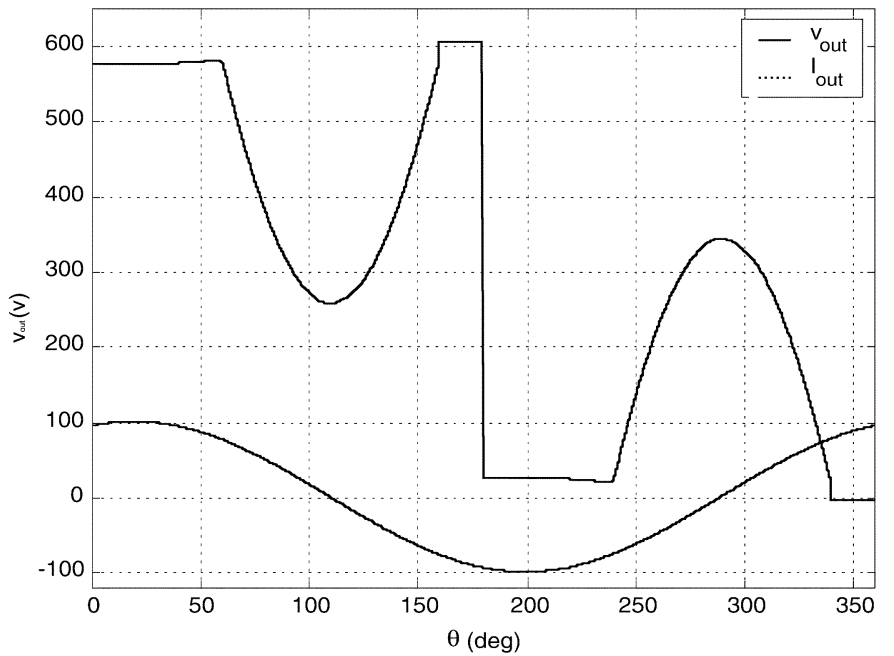

Fig. 17. High loss during incorrect commutation.

During normal operation, the resonant circuit is excited such that it appears predominantly inductive (to reduce commutation losses). However, if the unmodified describing function is coupled to a transient FMA model of a high-order resonant circuit (such as an in-circuit averaged model), the resulting system model can exhibit instability if the current transiently assumes a capacitive characteristic. Use of describing-functions derived from (24)-(26) address this issue. Moreover, if $\theta_{d}$ becomes greater than $\theta_{2}$ (a highly inductive characteristic), the diode will still be conducting when the switch turns off, implying that the capacitors do not begin to support the current until $I_{\text {out }}$ crosses zero (at $\theta_{d}$ ), thereby effectively limiting $\theta_{2}$ to $\geq \theta_{d}$. [10]

\section{CONCLUSION}

The paper presents the derivation of a novel describing function to model the output voltage of a H-bridge inverter, and includes a functional description of the relationship between the output- and supply-currents. Accuracy of the resulting model is demonstrated by comparison with SPICE simulation results, and with practical measurements from a prototype induction heating system. The model facilitates system simulation over a number of cycles of the input utility supply, ultimately allowing optimization of control systems without the significant computational overhead normally incurred by having to employ at switching-cycle level simulation. In particular, it is notable that the proposed model executes, typically, some 10000 times faster than a H-bridge inverter modeled using Spice.

Limitations of model applicability are discussed, with particular emphasis to operation during incorrect commutation of the $\mathrm{H}$-bridge, along with suggested modifications to the proposed model where appropriate.

\section{REFERENCES}

[1] K. Chen and T. A. Stuart, "A study of IGBT turn-off behavior and switching losses for zero-voltage and zero-current switching," in Proc. 7th Annu. Applied Power Electronics Conf. Expo., New York, 1992, pp. 411-418.
[2] E. J. Dede, V. Esteve, E. Maset, J. M. Espi, A. E. Navarro, J. A. Varrasco, and E. Sanchis, "Soft switching series resonant converter for induction heating applications," in Proc. Int. Conf. Power Electronics and Drive Systems, vol. 2, 1995, pp. 689-693.

[3] N. Frohleke, J. Kunze, A. Fiedler, and H. Grotstollen, "Contribution to the AC-analysis of resonant converters; analysis of the series-parallel resonant converter including effects of parasitics and lossless snubber for optimized design," in Proc. 7th Annu. Applied Power Electronics Conf. Expo. (APEC'92), 1995, pp. 219-228.

[4] University of California Berkley Spice, Standard 3f3, 2003

[5] SIMULINK, Mathworks Inc., 2003.

[6] SABER, Std. v5.1, 2003.

[7] Data Sheet (2003). Standard APT5010LVR [Online]. Available: www.advancedpower.com

[8] A. D. Pathak, "MOSFET/IGBT drivers theory and applications," IXYS Applicat. Note AN0002, IXYS Corporation, Santa Clara, CA, 2003.

[9] International Rectifier, IRFPS40N50L data sheet www.irf.com, 2003.

[10] H. I. Sewell, D. A. Stone, and C. M. Bingham, "Novel, three phase, unity power factor modular induction heater," Proc. Inst. Elect. Eng. B., vol. 147, no. 5, pp. 371-378, Sept. 2000.

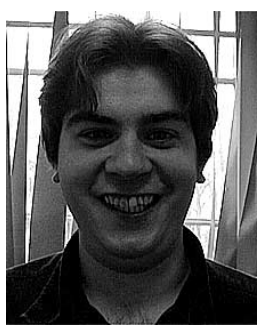

H. Isaac Sewell received the M.Eng. degree in electronic and electrical engineering and the Ph.D. degree from the University of Sheffield, Sheffield, U.K., in 1996 and 2002, respectively.

Since 2000, he has worked in industry as a Design Engineer at Inductelec, Ltd., Sheffield, and as a Research Associate in the Department of Electronic and Electrical Engineering, University of Sheffield, where his research interests include induction heating, mains supply power factor correction, and analysis of resonant power converters.

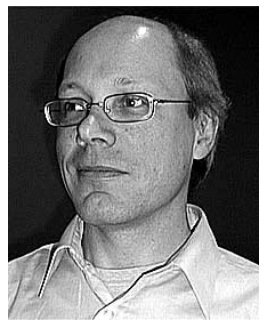

David A. Stone received the B.Eng. degree in electronic engineering from the University of Sheffield, Sheffield, U.K., in 1984 and the Ph.D. degree from Liverpool University, Liverpool, U.K., in 1989.

He then returned to the University of Sheffield as a Member of Academic Staff specializing in power electronics and machine drive systems. His current research interests are in resonant power converters, hybrid-electric vehicles, battery charging, EMC, and novel lamp ballasts for low pressure fluorescent lamps.

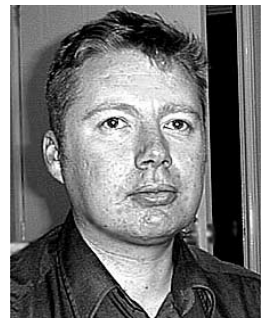

Chris M. Bingham (M'94) received the B.Eng. degree in electronic systems and control engineering, from Sheffield City Polytechnic, Sheffield, U.K., in 1989, the M.Sc. degree in control systems engineering from the University of Sheffield, in 1990 and the Ph.D. degree from Cranfield University, Cranfield, U.K., in 1994. His Ph.D. research was on control systems to accommodate nonlinear dynamic effects in aerospace flight-surface actuators.

He remained with Cranfield University as a PostDoctoral Researcher, until subsequently taking up a research position at the University of Sheffield. Since 1998, he has been a Lecturer in the Department of Electronic and Electrical Engineering, University of Sheffield. His current research interests include traction control/antilock braking systems for electric vehicles, electromechanical actuation of flight control surfaces, control of active magnetic bearings for high-speed machines, and sensorless control of brushless machines. 